\title{
Hepatic Steatosis Markers in Diabetic Rats Trained at the Aerobic/
} Anaerobic Transition

\section{Leandro Pereira de Moura ${ }^{1 *}$, Ricardo José Gomes ${ }^{2}$, José Alexandre Curiacos de Almeida Leme ${ }^{1}$, Michel Barbosa de Araújo ${ }^{1}$ and Maria} Alice Rostom de Mello ${ }^{1}$

${ }^{1}$ São Paulo State University, Physical Education Departament- Rio Claro/SP, Brazil

${ }^{2}$ Universidade Federal de São Paulo, Departamento de Educação Física- Santos/SP, Brazil

\begin{abstract}
Aims: To analyze the effects of exercise performed at the aerobic/anaerobic transition (Lan) on Non-Alcoholic Hepatic Steatosis (NAHS) markers in type 1 diabetic rats.

Methods: Adult Wistar rats were separated into four groups: Sedentary Control (SC), Trained Control (TC), Sedentary Diabetic (SD) and Trained Diabetic (TD). The trained groups swam supporting workloads equivalent to the Lan for one hour/day, five days/week, for eight weeks. Body weight, serum albumin concentrations, glucose concentrations, Free Fatty Acid (FFA) concentrations, NAHS markers (Aspartate Aminotransferase (AST) and Alanine Aminotransferase (ALT) and total lipid concentrations in the liver were analyzed.

Results: The diabetic groups showed higher serum glucose concentrations and more weight loss compared to the controls, although the TD group was less affected than the SD group. Training resulted in a decrease in serum glucose levels in the diabetic rats. The NAHS markers, total lipid concentrations in the liver and serum albumin concentrations did not differ between the groups. However, the diabetic animals had higher serum FFA levels than the controls.

Conclusion: Physical training at Lan attenuates weight loss and improves serum glucose homeostasis in diabetic animals. In addition, serum ALT and AST enzymes proved to be adequate markers of lipid levels in the livers in this animal model.
\end{abstract}

Keywords: Diabetes mellitus; Non-alcoholic hepatic steatosis; Physical training

\section{Introduction}

Diabetes Mellitus (DM) results from a reduction in either the secretion or the action of insulin and causes impairment in both glucose storage and glucose use by cells. According to World Health Organization (WHO), there were approximately 170 million people with diabetes around the world in 2002, and it has been predicted that by the year 2030 this number may double [1].

Type 1 Diabetes Mellitus (DM1) can result in serious complications that reduce both quality of life and life expectancy [2]. These complications include retinopathy, renal disease, atherosclerotic macrovascular disease, debilitating neuropathies and hepatic diseases, such as Hepatic Steatosis (HS).

HS is characterized by an accumulation of fat in the liver that exceeds $5 \%$ of the total weight [3] and can result from alcohol consumption or other factors [4]. Non-Alcoholic Hepatic Steatosis (NAHS) is one of the most common causes of chronic hepatic diseases, followed by hepatitis $\mathrm{C}$ and alcoholic HS.

DM1 can lead to an imbalance of the lipid profile [5,6]. According to Carew et al. [7], the imbalance of serum lipoprotein concentrations promotes clearance of cholesterol from peripheral tissues to the liver for catabolism and excretion. When transfer to the liver is high and the rate of catabolism is low, fat accumulation may occur in this organ, resulting in NAHS. Markers of this condition, such as rises in the serum concentrations of the hepatic transaminases Aspartate Aminotransferase (AST) and Alanine Aminotransferase (ALT), are used as indicators of DM in epidemiological studies [8].

Many attempts have been made to develop drugs that target the symptoms of NAHS. However, due to the lack of drugs that treat NAHS specifically, patients are instructed to both reduce the amount of lipids and carbohydrates in their diet and practice regular physical activity to increase their daily energy expenditure [9]. Physical exercise is considered to be of great importance in the treatment of DM [10]. Such intervention is known to improve glucose tolerance and lipid profiles because muscle contraction activates the insulin signaling pathway $[11,12]$ and promotes glucose uptake, even in the absence of insulin, therefore improving glucose and lipid metabolism. However, there is controversy concerning the most effective exercise protocols for diabetic patients. In a study performed by Harrison and Neuschwander-Tetri [13], exercise training resulted in gradual and healthy weight loss, as well as an improvement of diabetic NAHS symptoms from a histological point of view. However, in other studies performed by the same authors, sharp weight loss resulted in a worsening of the clinical condition of the patients, predisposing them to hepatic fibrosis. Therefore, the present study aims to analyze the effects of physical exercise an intensity equivalent to the aerobic/anaerobic metabolic transition on NAHS markers in type 1 diabetic rats.

\section{Material and Methods}

\section{Animals}

Young male Wistar rats (60 days old at the start of the experiment

${ }^{*}$ Corresponding author: Leandro Pereira de Moura, São Paulo State University, Physical Education Department, Rio Claro, SP, Brazil, Tel: +55(19)8197-9999; E-mail: leandropereiram@hotmail.com

Received October 30, 2013; Accepted November 29, 2013; Published Decembe 07, 2013

Citation: de Moura LP Gomes RJ, de Almeida Leme JAC, de Araújo MB, de Mello MAR (2013) Hepatic Steatosis Markers in Diabetic Rats Trained at the Aerobic/ Anaerobic Transition. J Liver 2: 137. doi:10.4172/2167-0889.1000137

Copyright: (C) 2013 de Moura LP, et al. This is an open-access article distributed under the terms of the Creative Commons Attribution License, which permits unrestricted use, distribution, and reproduction in any medium, provided the original author and source are credited. 
(300g), 10 per group) were obtained from the animal facilities of UNESP - Sao Paulo State University, Botucatu, SP, Brazil. During the experiment, the rats had free access to food (Purina ${ }^{\circ}$ balanced chow) and water and were housed (5 rats per cage) on a 12-h light/dark cycle at a temperature of $21^{\circ} \mathrm{C}$. All of the procedures involving animals were approved by the Institutional Committee for Ethics in Animal Research (CEUA-IB-UNESP/Rio Claro, Protocol number: 008/2009).

\section{Induction of diabetes}

To induce experimental diabetes, the rats received monohydrated alloxan (from Sigma, $32 \mathrm{mg} / \mathrm{kg}$ of body weight) dissolved in $0.01 \mathrm{M}$ citrate buffer, $\mathrm{pH}$ 4.5. The rats were fasted for one night and then injected in the penile dorsal vein [14]. After this procedure, the rats were placed in collective cages ( 5 rats per cage) where they received a solution of water and glucose (15\%) for the first 24 hours post-alloxan injection to avoid the complications of alloxanic hypoglycemia [15]. Five days after the administration of the drug, the serum glucose levels of the rats in the fed state were measured. Rats that were not diabetic $(<250 \mathrm{mg} / \mathrm{dL})$ or that were extremely diabetic $(>650 \mathrm{mg} / \mathrm{dL})$ were excluded from the study. Rats injected with a vehicle (citrate buffer) were used as controls.

\section{Experimental groups and design}

The animals were divided into four groups containing 10 animals each: Sedentary Control (SC) - healthy rats that did not perform physical exercise; Trained Control (TC)-healthy rats that performed a protocol of physical exercise; Sedentary Diabetic (SD)-alloxanic diabetic rats that did not perform physical exercise and Trained Diabetic (TD) alloxanic diabetic rats that performed a protocol of physical exercise.

\section{Acclimation to the water}

All of the rats were acclimated to the water before the beginning of the exercise tests. The acclimation involved keeping the animals in shallow water maintained at $31 \pm 1^{\circ} \mathrm{C}$ for $60 \mathrm{~min}, 5$ days/week, for 2

\section{Lactate Knectic}

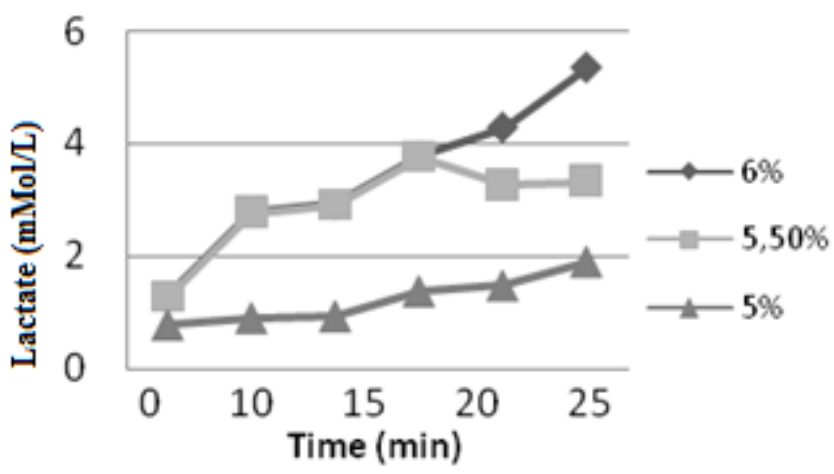

Figure 1: The blood lactate concentration of one representative rat during the swimming tests that were used to identify the Maximal Lactate Steady State (MLSS). The animal was subjected to three tests while supporting workloads of $5.0 \%, 5.5 \%$ and $6.0 \%$ of his body weight. Each test consisted of a continuous swimming exercise at the intensity previously established for 25 min or until exhaustion. Blood was collected at rest and at 5-min intervals during exercise. MLSS was defined as the highest workload at which the blood lactate concentration did not vary by more than $1 \mathrm{mmol} / \mathrm{L}$ between 10 and 20 min of exercise. For this specific rat, the MLSS was reached while supporting a load of $5.5 \%$ of his body weight at a blood lactate concentration of $3.34 \pm 0.60$. weeks. The purpose of acclimating the rats was to reduce stress without allowing the rats to adapt to the exercise training [16].

\section{Aerobic/anaerobic metabolic transition and aerobic conditioning}

Immediately following the induction of diabetes, all of the animals were subjected to an effort trial to determine the individual exercise intensity required to reach the aerobic/anaerobic metabolic transition, according to the Maximal Lactate Steady State (MLSS) protocol. This method was designed to detect the highest blood lactate concentration at which the entrance of lactate into the blood stream was counterbalanced by its removal, thereby maintaining a stable concentration during exercise at a constant intensity [17]. This method has proven to be useful for recommending exercises and for determining the aerobic capacity of humans, rats and mice [18-20]. Our research group designed the MLSS protocol for rats [21] that was used for the swimming exercises in this study. In short, to determine the MLSS, a series of $25 \mathrm{~min}$ swimming exercises were performed in which the rats supported increasing workloads in relation to body weight (starting at $5 \%$ and increasing in $1 \%$ increments after each test). The workload was fixed for each series, and there was a 48 -h interval between each exercise. Blood samples $(25 \mu \mathrm{L})$ were taken from a cut on the tail tip every $5 \mathrm{~min}$ for determination of lactate levels. The concentration at the highest workload in which the blood lactate concentration did not vary by more than $1.0 \mathrm{mmol} / \mathrm{L}$ between 10 and $25 \mathrm{~min}$ of exercise [21,22] was considered to be the blood lactate concentration equivalent to the MLSS. The blood lactate concentration was determined using the enzymatic method proposed by Engels and Jones [23] (Figure 1).

\section{Exercise protocol}

The trained animals were subjected to swimming exercise for 1 $\mathrm{h} /$ day, 5 days/week, supporting a workload equivalent to the aerobic/ anaerobic metabolic transition, as estimated by the MLSS tests [21].

\section{General evaluations and evaluation of hepatic steatosis} markers

Serum glucose levels (Laborlab Kit, Guarulhos-SP/Brazil) were analyzed at the beginning and end of the experiment, and the animals were weighed once a week. For analysis of body weight during the eightweek experiment, total area under the curve was calculated using the trapezoidal method [24] with the aid of the Origin $6.0^{\circ}$ software. All of the animals were killed by decapitation 48 hours after the last exercise session, and the blood was collected for serum hepatic Aspartate Aminotransferase (AST), Alanine Aminotransferase (ALT), Free Fatty Acid (FFA) and albumin measurements using commercially available kits (Laborlab Kit, Guarulhos-SP/Brazil). Liver samples were collected for determination of total lipid concentrations [25].

\section{Statistic Analysis}

The results are presented as mean \pm standard deviation and were analyzed by one-way ANOVA, followed by the Bonferroni post-hoc test in appropriate cases. Statistica $7.0^{\circ}$ software was used to perform the analyses, and p-values less than 0.05 were considered significant.

\section{Results}

The serum glucose concentrations of the diabetic animals were increased compared to the controls and a significant decrease was observed in the Trained Group (TD) when compared to the Sedentary Group (SD) (Table 1). Serum albumin concentrations did not differ between the groups (Table 1). 


\begin{tabular}{|c|c|c|c|} 
& TC & SC & D \\
\hline Serum Albumin $(\mathbf{g} / \mathbf{d L})$ & $4.3 \pm 0.5$ & $40.2 \pm 0.4$ & $4.0 \pm 0.5$ \\
\hline Serum Glucose $(\mathbf{m g} / \mathbf{d L})$ & $105.0 \pm 9.4$ & $102.7 \pm 13.2$ & $301.4 \pm 13.9^{* *}$ \\
\hline Serum FFA $(\boldsymbol{\mu E q} / \mathbf{L})$ & $432.0 \pm 41.3$ & $449.3 \pm 20.8$ & $323.2 \pm 12.1^{*}$ \\
\hline Body Weight $(\mathbf{g}$ × $\mathbf{8}$ weeks) & $5383.5 \pm 314.9$ & $5270.7 \pm 198.3$ & $507.8 \pm 30.0^{*}$ \\
\hline
\end{tabular}

$\mathrm{TC}=$ Trained Control, $\mathrm{SC}=$ Sedentary Control, TD=Trained Diabetics, SD=Sedentary Diabetics

* Different from control groups (ANOVA, $p<0.05)$.

${ }^{* *}$ Different from SD (ANOVA, $\left.p<0.05\right)$.

Table 1: Serum albumin, glucose and FFA concentrations at the end of the experiment and area under the curve calculations for body weight during the experiment.

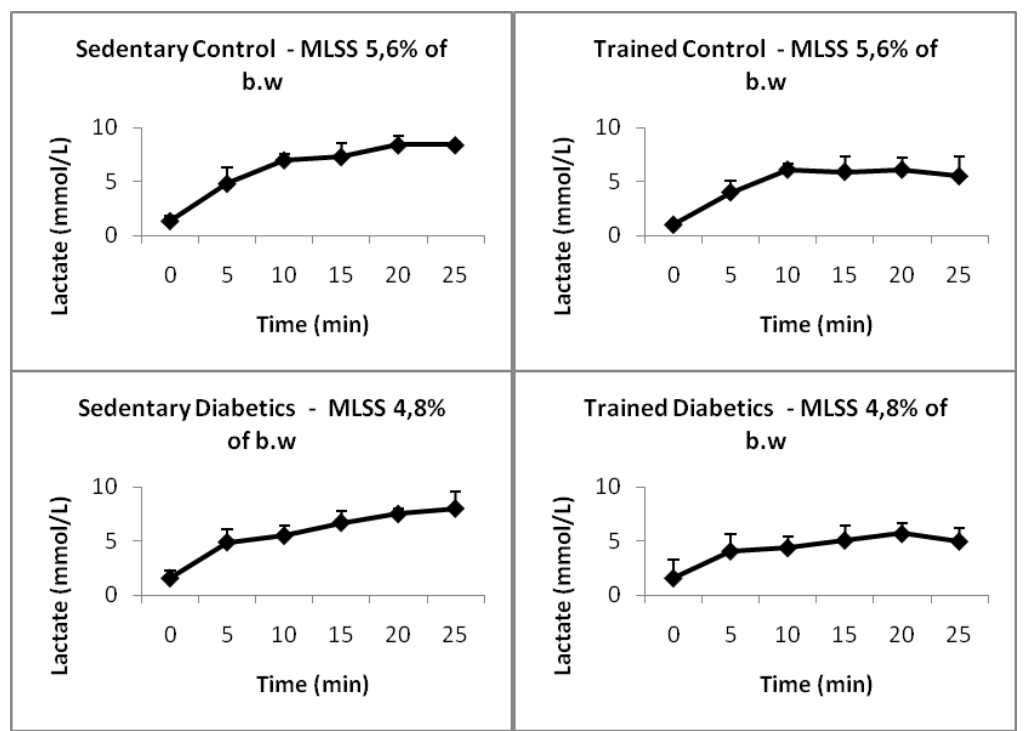

Figure 1: Blood lactate kinetics during swimming exercise at the MLSS-equivalent intensity. Data are expressed as mean \pm standard deviation of 10 rats per group. The workload equivalent to the MLSS in percentage of body weight is shown at the top of each panel.

\begin{tabular}{|c|c|c|c|}
\hline & TC & SC & TD \\
\hline Serum AST (U/L) & $87.0 \pm 23.2$ & $81.6 \pm 15.0$ & $78.7 \pm 29.9$ \\
\hline Serum ALT (U/L) & $38.9 \pm 9.4$ & $39.7 \pm 14.6$ & $44.6 \pm 17.0$ \\
\hline Total liver lipid concentrations $\mathbf{( m g / 1 0 0 ~} \mathbf{~ m g )}$ & $3.5 \pm 0.5$ & $3.5 \pm 0.4$ & $40.0 \pm 17.4$ \\
\hline
\end{tabular}

TC= Trained Control, SC= Sedentary Control, TD= Trained Diabetics, SD= Sedentary Diabetics

Table 2: Markers of non-alcoholic hepatic steatosis at the end of the experiment.

We were able to identify the individual MLSS for each animal. No differences in blood lactate concentration at the intensity equivalent to aerobic/anaerobic metabolic transition, as determined by the MLSS test, were observed between the groups. However, the MLSS-equivalent workload of the diabetic groups was lower than that of the control groups (Figure 2).

The body weights of the animals were measured weekly, and the area under the curve was calculated for each animal. The diabetic groups showed a significant reduction in body weight compared to the control groups, although the TD group was less affected than the SD group (Table 1).

Total lipid concentration in the liver, as well as serum AST and ALT activity, did not differ between the groups (Table 2). However, serum FFA levels were increased in the diabetic groups compared to the control groups (Table 1).

\section{Discussion}

In the present study, a condition similar to human type 1 diabetes was induced in rats by alloxan-injection, which resulted in both hyperglycemia and a reduction of body mass, that were similar to the effects observed in previous studies conducted by Luciano and Lima [14]. Serum albumin concentrations were measured to assess the hydration state of the animals. An increase in albuminemia is commonly observed in the dehydrated state and is caused by the excessive loss of water or water privation, which generates hemo concentration [26]. No differences were observed between the groups in the present study, indicating that the diabetic rats were not dehydrated.

At the beginning of the experiment, the MLSS swimming test was performed to determine the exercise intensity equivalent to the aerobic/ anaerobic metabolic transition. The analysis of the blood lactate kinetics during the swimming exercise allowed us to determine the workload that was equivalent to the MLSS intensity in the control and diabetic rats. Gobatto et al., [21] reported that the MLSS in eutrophic rats occurred at a mean blood lactate concentration of $5.5 \mathrm{mmol} / \mathrm{L}$ when the animals carried workloads that were equivalent to 5\%-6\% of their body weight. Similar values were observed in the control rats of the present study. However, the workload equivalent to the MLSS intensity was lower in the diabetic rats compared to the control rats, 
Citation: de Moura LP, Gomes RJ, de Almeida Leme JAC, de Araújo MB, de Mello MAR (2013) Hepatic Steatosis Markers in Diabetic Rats Trained at the Aerobic/Anaerobic Transition. J Liver 2: 137. doi:10.4172/2167-0889.1000137

indicating that the aerobic capacities of the diabetic rats were impaired. During exercise at MLSS intensity, control and diabetic rats had similar physiological responses when blood lactate kinetics was analyzed.

The weight loss of diabetic groups was due to the insulin deficit, as this hormone is responsible for anabolism and plays important roles in carbohydrate, amino acid and fat metabolism [27,28]. Exercise training may attenuate body weight loss, since physical activity increases insulin sensitivity and decreases serum glucose concentrations [29,30]. In the present study, we observed less weight loss in the DT group than the DS group.

Another aspect to be highlighted is that, even though the training protocol used did not result in a reduction of the diabetes-induced increase in serum FFAs, it did improve glycemic control in the animals. It is known that physical exercise increases insulin sensitivity, and therefore improves glucose uptake [31]. A chronic effect of this practice is an increase in the sensitivity of cells to insulin, which increases the effects of this hormone on the phosphorylation of substrate- 2 of the insulin receptor (IRS-2) and the phosphatidylinositol-3-kinase (PI3K) activity [31]. When muscle contractions take place, there are increases in both mitogen-activated protein kinase enzyme activity and AMP activated protein kinase activity, followed by increases in calcium and nitric oxide levels. These events promote the translocation of vesicles containing GLUT-4 to the periphery of the cells, thus allowing glucose to enter the muscle fibers [32].

There were no differences in total lipid content in the liver between the control and diabetic groups. There were also no differences in the levels of the NAHS marker enzymes AST and ALT. Diabetic individuals who are insulin resistant tend to develop NAHS due to a greater activation of lipases, followed by an increase in the transport of fatty acids to the liver, which leads to a significant uptake of triglycerides by hepatocytes [33]. The NAHS was not observed in the present study due, at least in part, to the fact that the FFAs were probably previously consumed as an energy substrate because the animals had uncompensated diabetes. Further studies of liver metabolism in this type 1 diabetic rat model are required.

In summary, exercise training at the aerobic/anaerobic metabolic transition attenuates body weight loss and reduces serum glucose concentrations in diabetic rats. Total lipids in the liver, as well as serum NAHS markers, were not affected by either alloxan-induced diabetes or exercise training.

\section{Conclusions}

The results suggest that physical training at an intensity equivalent to the metabolic transition attenuates body weight loss and improves serum glucose homeostasis in diabetic animals. In addition, serum ALT and AST proved to be adequate markers of liver lipid levels in this animal model.

\section{Acknowledgement}

The authors would like to thank Clarice Y. Sibuya, Eduardo Custódio and José Roberto R. Silva for technical assistance. This research was supported by the Brazilian foundations FAPESP, CNPq and CAPES.

\section{References}

1. Wild S, Roglic G, Green A, Sicree R, King H (2004) Global prevalence of diabetes: estimates for the year 2000 and projections for 2030. Diabetes Care 27: 1047-1053

2. M A Kelly, M L Rayner, C H Mijovic, A H Barnett (2003) Molecular aspects of type 1 diabetes. J. Clin. Pathol 56: 1-10.
3. Leclercq I, Horsmans Y, Desager JP, Delzenne N, Geubel AP (1998) Reduction in hepatic cytochrome P-450 is correlated to the degree of liver fat content in animal models of steatosis in the absence of inflammation. J. Hepatol 28 410-416.

4. Alba LM, Lindor K (2003) Review article: non-alcoholic fatty liver disease Aliment Pharmacol Ther 17: 977-86.

5. Colca JR, Dailey CF, Palazuk BJ, Hillman RM, Dinh DM, et al. (1991) Pioglitazone hydrochloride inhibits cholesterol absorption and lowers plasma cholesterol concentrations in cholesterol-fed rats. Diabetes 12: 1669-1674.

6. Sharma RD, Raghuram TC (1990) Hypoglycemic effect of fenugreek seeds in noninsulin dependent diabetic subject. Nutr Res 10: 731-739.

7. Carew TE, Koschinsky T, Hayes SB, Steinberg D (1976) A mechanism by which high-density lipoproteins may slow the atherogenic process. Lancet 1: 1315 1317.

8. Mukai M, Ozasa K, Hayashi K, Kawai K (2002) Various S-GOT/S-GPT ratios in nonviral liver disorder and related physical conditions and life style. Digestive Disorders and Sciences 47: 549-555.

9. Ten S, Malaren N (2003) Insulin resistance syndrome in children. J Clin Endocrinol Metab 89:2596-2539.

10. American Diabetes Association (2006) ADA stand position: physical activity/ exercise and diabetes mellitus. Diabetes Care 26: 573-577.

11. Cortright RN, Dohm GL (2006) Mechanisms by which insulin and contraction stimulate glucose transport. Can. J Appl Physiol 22: 519-530.

12. Luciano E, Carneiro EM, Carvalho CR, Carvalheira JB, Peres SB, et al. (2002) Endurance training improves responsiveness to insulin and modulates insulin signal transduction through the phosphatidylinositol 3-kinase/Akt-1 pathway. Eur J Endocrinol 147: 149-157.

13. Harrison S, Neuschwander-Tetri B (2004) Nonalcoholic fatty liver disease and nonalcoholic steatohepatitis. Clinics in Liver Disease 8: 861-879.

14. Luciano E, Lima FB (1997) Metabolismo de ratos diabéticos treinados submetidos ao jejum e ao exercício. Revista de Ciências Biomédicas 18: 4760.

15. Lenzen S (2008) The mechanisms of alloxan- and streptozotocin-induced diabetes. Diabetologia 2: 216-226.

16. Voltarelli FA, Gobatto CA, Mello MAR (2002) Determination of anaerobic threshold in rats using the lactate minimum test. Braz. J Med Biol Res 35 1389-1394.

17. Heck H, Mader A, Hess G, Mücke S, Müller R, et al. (1985) Justification of the 4- $\mathrm{mmol} / \mathrm{l}$ lactate threshold. Int J Sports Med 6: 117-130.

18. Beneke R, Leithäuser RM, Hütler M (2001) Dependence of the maximal lactate steady state on the motor pattern of exercise. Br J Sports Med 35: 192-196.

19. Contarteze RV, Manchado Fde B, Gobatto CA, De Mello MA (2008) Stress biomarkers in rats submitted to swimming and treadmill running exercises. Comp. Biochem. Physiol. Mol Integr Physiol 151: 415-422.

20. Ferreira JC, Rolim NP, Bartholomeu JB, Gobatto CA, Kokubun E, et al. (2007) Maximal lactate steady state in running mice: effect of exercise training. Clin Exp Pharmacol Physiol 34: 760-765.

21. Gobatto CA, de Mello MA, Sibuya CY, de Azevedo JR, dos Santos LA, et al. (2001) Maximal lactate steady state in rats submitted to swimming exercise. Comparative Biochemistry and Physiology. Comp Biochem Physiol Mol Integr Physiol 130: 21-27.

22. Beneke R (2003) Methodological aspects of maximal lactate steady stateimplications for performance testing. Eur J Appl Physiol 89: 95-99.

23. Engels RC, Jones JB (1978) Causes and elimination of erratic blanc in enzymatic metabolic assays involving the use of NAD in alkaline hydrazine buffers: improved conditions for assay of L-glutamate, L-lactate and other metabolites. Anal Biochem 88: 475-484

24. Matthews JN, Altman DG, Campbell MJ, Royston P (1990) Analysis of serial measurements in medical research. BMJ 300: 230-235.

25. Nogueira AR, Alves PM, Miranda RF, Boechat NL (1990) Colesterol e outros fatores de risco cardiovasculares nos servidores da UFRJ. Prevalência e influência de variáveis. Arq Bras Cardiol 55: 227-232.

26. Peters T, Biamont GT, Doumas BT (1982) Albumin in serum Em Faulkner WR Meites S, eds Selected Methods Clin Chem 9:319. 
Citation: de Moura LP, Gomes RJ, de Almeida Leme JAC, de Araújo MB, de Mello MAR (2013) Hepatic Steatosis Markers in Diabetic Rats Trained at the Aerobic/Anaerobic Transition. J Liver 2: 137. doi:10.4172/2167-0889.1000137

Page 5 of 5

27. American Diabetes Association Diagnosis and classification of Diabetes Mellitus (2006) Diabetes Care 29: 43-48.

28. Guyton AC, Hall JE (2006) Tratado de Fisiologia Médica. Rio de Janeiro, RJ: Guanabara Koogan.

29. Aronson D, Violan M A, Dufresne S D, Zangen D, Fielding R A, et al. (1997) Exercise stimulates the mitogen-activated protein kinase pathway in human skeletal muscle. J Clin Invest 99: 1251-1257

30. Hardin DS, Azzarelli B, Edwards J, Wigglesworth J, Maianu L, et al. (1995) Mechanisms of enhanced insulin sensitivity in endurance-trained athletes: effects on blood flow and differential expression of GLUT 4 in skeletal muscles. $\mathrm{J}$ Clin Endocrinol Metab 80: 2437-2446.
31. Howlett KF, Sakamoto K, Hirshman MF, Aschenbach WG, Dow M, et al. (2002) Insulin signaling after exercise in insulin receptor substrate-2-deficient mice. Diabetes 51: 479-483.

32. Jessen N, Goodyear LJ (2005) Contraction signaling to glucose transport in skeletal muscle. J Appl Physiol 99: 330-337.

33. Sass DA, Chang P, Chopra KB (2005) Nonalcoholic fatty liver disease: a clinica review. Digest Dis Sci 50: 171-180. 